\title{
Diseño de un Interferómetro Heterodino de Microondas para la Medición de la Densidad Electrónica del Plasma en el SCR-1
}

Jorge Sánchez-Castro ${ }^{1}$, Ivan Vargas²

Sánchez-Castro, J; Vargas, I. Diseño de un Interferómetro Heterodino de Microondas para la Medición de la Densidad Electrónica del Plasma en el SCR-1. Tecnología en Marcha.

Vol. 33, especial Movilidad estudiantil. Octubre, 2020. Pág 4-10.

doi) https://doi.org/10.18845/tm.v33i7.5470 


\section{Palabras clave}

Interferómetro; Heterodino, SCR-1; Densidad Electrónica; Microondas; PLL; Stellarator; Frecuencia.

\section{Resumen}

El Stellarator de Costa Rica 1 (SCR-1) es un dispositivo pequeño de confinamiento magnético de tipo stellarator modular que se emplea para la investigación de plasmas de alta temperatura y baja densidad, el primero de América Latina. [1]. El propósito de este trabajo es diseñar un dispositivo capaz de medir experimentalmente, de forma precisa, la densidad electrónica del plasma. Es por esto que se pretende diseñar un interferómetro heterodino de microondas, uno de los primeros diagnostico experimentales que se utilizan para medir este parámetro, debido a su alta fiabilidad. [2] En este documento, se calcula la frecuencia optima de operación, junto con su respectivo desfase máximo. Además, se presenta un diagrama de bloques del sistema, que da hincapié a la especificación de cada uno de los componentes del diseño y a su vez sera soporte para realizar un balance de potencia y un análisis del comportamiento teórico de la señal a lo largo del sistema. Se concluyo que el sistema cumple con los requerimientos necesarios para su implementación.

\section{Introducción}

El 29 de junio de 2016, el Stellarator de Costa Rica 1 (SCR1) produjo su primer plasma de hidrógeno, convirtiéndose en uno de los pocos Stellarator operativos en el mundo. [3] Hito que dio paso a la necesidad de implementar diferentes diagnósticos que contribuyan con la caracterización del plasma de alta temperatura (15eV) y baja densidad $\left(7,4510^{16} \mathrm{~m}^{-3}\right)$ confinado en este dispositivo. Actualmente el proyecto cuenta con un Espectrometro óptico, una Sonda Langmuir y un Sistema de Mapeo Magnético como diagnósticos experimentales operativos.

Por otra lado, la interferometría de ondas electromagnéticas es uno de los primeros diagnósticos que se utilizan para medir la densidad electrónica de plasma, dado a su alta fiabilidad; [2]. Esta técnica se basa en el principio de superposición de dos o mas ondas coherentes (con amplitud y fase iguales) que se propagan por diferentes medios, desde su fuente hasta su punto de detección. El estudio de las alteraciones que sufren estas señales al propagarse, permite determinar parámetros físicos del medio. [4]

Un interferómetro esta compuesto por tres elementos principales: un oscilador, que permita generar una señal de entrada; un acople direccional, que divide esta señal en dos caminos diferentes (un camino de referencia y otro que sera sometido al medio de estudio); y un mezclador (detector) que da como resultado una función dependiente de ambas entradas. [3] Dependiendo del diseñador y del tipo de interferómetro se le agregan otros componentes como guías de onda, antenas, aisladores, desfasadores, atenuadores; que no solo mejoran la exactitud de la medición, sino que también cumplen funciones de protección contra potencias reflejadas.

\section{Cálculos previos}

Frecuencia de operación

Al diseñar un interiormente uno de sus factores por definir es su frecuencia de operación. Esta se calcula mediante la utilización de los conceptos de densidad de corte $\left(n_{c}\right)$ y densidad electrónica esperada $\left(\mathrm{n}_{\mathrm{e}}\right)$, que se relacionan matemáticamente de la forma: 


$$
\mathrm{n}_{\mathrm{c}}=\mathrm{A} \cdot \mathrm{n}_{\mathrm{e}}
$$

Según [5], Un buen criterio de diseño es lograr que la densidad de corte sea 3 o 4 veces mayor que la mayor densidad electrónica esperada.Por otro lado, la ecuación 13 referida de [6]; permite obtener el índice de refracción para una onda. Con la expresión:

$$
\mathrm{N}^{2}=1-\frac{\mathrm{w}_{\mathrm{pe}}^{2}}{\mathrm{w}^{2}}
$$

donde $\mathrm{w}_{\mathrm{pe}}$ es la frecuencia de oscilación del plasma, dada por:

$$
\mathrm{w}_{\mathrm{pe}}=1-\frac{\mathrm{n}_{\mathrm{e}} \mathrm{e}^{2}}{\varepsilon_{0} \mathrm{~m}_{\mathrm{e}}}
$$

La densidad crítica de electrones se obtiene cuando $\mathrm{N}=0$, es decir,cuando ocurre un corte en el plasma (donde se da la reflexión de la onda), por lo que al sustituir (2) en (3), se tiene que:

$$
\mathrm{n}_{\mathrm{c}}=\frac{\varepsilon_{0} \mathrm{~m}_{\mathrm{e}} \mathrm{w}^{2}}{\mathrm{e}^{2}}
$$

La frecuencia angular de la onda la reescribimos en términos de la frecuencia y tenemos que:

$$
\mathrm{n}_{\mathrm{c}}=\frac{4 \pi^{2} \varepsilon_{0} \mathrm{~m}_{\mathrm{e}}}{\mathrm{e}^{2}} \mathrm{f}^{2}
$$

Finalmente de (1) y (5) encontramos la frecuencia de operación definida como:

$$
\mathrm{f}=\left(\frac{\mathrm{e}^{2}}{4 \pi^{2} \varepsilon_{0} \mathrm{~m}_{\mathrm{e}}} \mathrm{n}_{\mathrm{e}}\right)^{\frac{1}{2}}
$$

Por lo tanto, al tener una densidad electrónica teórica (ne) de 7,45 × 10 ${ }^{16} \mathrm{~m}^{-3}$ en el SCR-1, el interferómetro debe tener una frecuencia de operación (FO) cercana a $5 \mathrm{GHz}$ (banda C).

\section{II-B. Desfase máximo}

El desfase máximo se definido, según [6], con la ecuación:

$$
\Delta \phi=\frac{\mathrm{w}}{2 \mathrm{cn}_{\mathrm{c}}} \int \mathrm{n}_{\mathrm{c}} \mathrm{dl}
$$

Al considerar la densidad constante a lo largo de todo el plasma y al sustituir en (7), la ecuación (5)

$$
\Delta \phi=\frac{\mathrm{e}^{2} \mathrm{n}_{\mathrm{e}} \Delta \mathrm{Z}}{4 \pi^{2} \varepsilon_{0} \mathrm{~m}_{\mathrm{e}} \mathrm{cf}}
$$

En el caso de que el haz de microondas atraviese la columna de plasma a lo largo de su diámetro menor, se tiene que $\Delta Z \approx 0,11 \mathrm{~m}$ y entonces el desfase sería aproximadamente de $1,7 \mathrm{rad} \approx 98$ o 


\section{Diseño del sistema}

Existen diferentes tipos sistemas de interferometría, algunos de ellos son: Interferómetro Básico, Detección por cuadratura, Sistema Homodino, Sistema Heterodino. Estos se ven diferenciados por su simplicidad, sensibilidad y ambigüedad. Según [4] el sistema heterodino es la forma mas confiable para determinar las alteraciones en la fase; por lo tanto, en este documento vamos a obviar los otros tres posibles diseño.

Un sistema de detección heterodino, se basa en la idea de permitir un cambio de la frecuencia de entrada (cambio de banda de FO). Esto se logra, al combinar la señal de entrada (RF) con una señal coherente en fase (LO), en un elemento no lineal que convierte las señales de entrada, de una frecuencia dada, en una señal de salida con una frecuencia intermedia (IF). [4] El objetivo principal es tener una señal de salida con una frecuencia mucho menor a la frecuencia de operación del interferómetro, para así facilitar el procesamiento de la misma.

El diagrama de bloques que presenta en la figura 1 representa al diseño propuesto.

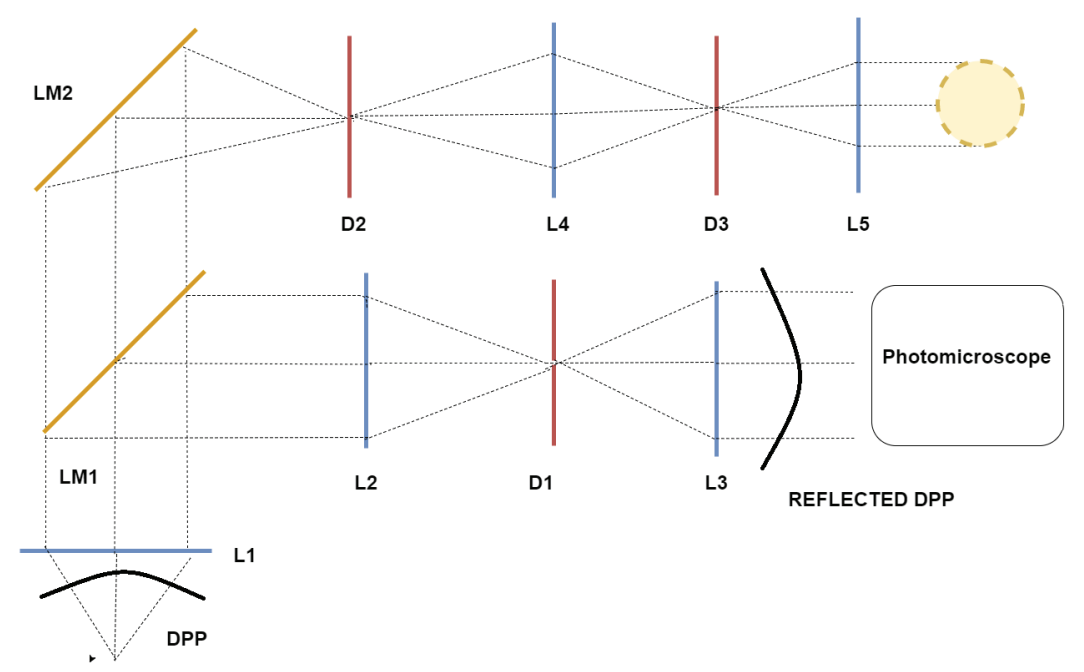

Figura 1: Diagrama de Bloques del Interferómetro Heterodino de Microondas Propuesto.

\section{A. Equipos y componentes}

Los osciladores locales se encargan de generar una señal de entrada de la forma Acos(wt); sin embargo, como ambos osciladores necesitan tener la misma fase, con el objetivo de simplificar el procesamiento de la señal de salida, se emplea un sistema PLL de referencia externa, para enlazar ambos osciladores. Además, estos deben de presentar una pequeña diferencia en su frecuencia de operación para no tener una salida completamente continua en los mezcladores. Específicamente para este diseño se empleara un PLL con las características mostradas en el cuadro 1.

Cuadro 1. Características del PLL.

\begin{tabular}{|l|c|}
\hline \multicolumn{1}{|c|}{ Parámetro } & Valor \\
\hline Frecuencia central & $5 \mathrm{GHz}$ \\
\hline Potencia & $25 \mathrm{dBm}$ \\
\hline Referencia & Externa $100 \mathrm{MHz} @ 0 \mathrm{dBm}$ \\
\hline
\end{tabular}


Los mezcladores son elementos no lineales que se encargan de convertir las señales de entrada RF y LO en un señal de frecuencia intermedia (IF). Específicamente, se utilizan mezcladores balanceados (equilibrados), donde se trabajara con el producto de intermodulación correspondiente a la resta resta de ambas señales. Las característica especificas de estos mezcladores se presentan en el cuadro 2.

Cuadro 2. Características del Mezclador.

\begin{tabular}{|l|c|}
\hline \multicolumn{1}{|c|}{ Parámetro } & Valor \\
\hline Calibración en RF & $-10 \mathrm{dBm}$ \\
\hline Potencia en LO & {$[11,15] \mathrm{dBm}$} \\
\hline
\end{tabular}

Se detallan únicamente las características y funcionamiento de los osciladores y mezcladores debido a su grado de importancia en el diseño. En el cuadro 3 se presenta la lista completa de componentes con su respectivo código de proveedor y una pequeña descripción del mismo, se excluyen las guías de onda.

Cuadro 3. Lista de equipos y componentes.

\begin{tabular}{|l|c|l|}
\hline \multicolumn{1}{|c|}{ Componente } & \multicolumn{1}{c|}{ Código } & \multicolumn{1}{c|}{ Descripción } \\
\hline Oscilador & QPL-0525ES & Oscilador tipo PLL \\
\hline Aislador & QIF-A00000 & Protección \\
\hline Mezclador & QMB-0528AS & Mezclador balanceado \\
\hline Acople direccional & QDC-EA1000 & Divisor de señales \\
\hline Antena & QRR-A00P30 & Antena piramidal \\
\hline Medidor de potencia & QEA-05FBAP & Protección \\
\hline Atenuador fijo & $\begin{array}{l}\text { QAF-A20000 } \\
\text { QAF-A04000 }\end{array}$ & $\begin{array}{l}\text { Atenuador de 20dB } \\
\text { Atenuador de 4dB }\end{array}$ \\
\hline
\end{tabular}

\section{B. Análisis del Comportamiento de la Señal}

Basándose en los puntos marcados en la figura 1, se analizara de forma teórica los procesos o cambios que sufre la señal a lo largo del circuito. Es así como, la señal que se propaga por el punto "A", tiene como objetivo servir de referencia para los PLL's y de tal forma que logran enlazar la fase de ambos osciladores.

$$
\mathrm{S}_{\mathrm{A}}=\mathrm{k}_{1} \cos \left(2 \pi \mathrm{f}_{1} \mathrm{t}\right)
$$

Donde $\mathrm{k}_{1}$ corresponde a la amplitud de la señal y $\mathrm{f}_{1}$ a su frecuencia $(\approx 100 \mathrm{MHz})$. En los punto $\mathrm{B}$ y $B^{\prime}$ se propagan las señales de entrada RF y LO respectivamente del primer mezclador, estas son las salidas directas del PLL.

$$
\begin{aligned}
& \mathrm{S}_{\mathrm{B}}=\mathrm{k}_{\mathrm{r}} \cos \left(2 \pi \mathrm{f}_{\mathrm{r}} \mathrm{t}\right) \\
& \mathrm{S}_{\mathrm{B}^{\prime}}=\mathrm{k}_{\mathrm{L}} \cos \left(2 \pi \mathrm{f}_{1} \mathrm{t}\right)
\end{aligned}
$$

Por otro lado, en el punto C, la señal ya se a propagado por el plasma ocasionado que esta sufra un desfase. 


$$
\mathrm{S}_{\mathrm{C}}=\mathrm{k}_{\mathrm{r}} \cos \left(2 \pi \mathrm{f}_{\mathrm{r}} \mathrm{t}+\phi\right)
$$

La ecuación (13) expresa la salida de los mezcladores.

$$
\begin{aligned}
& \mathrm{S}_{1}=\left(\mathrm{S}_{\mathrm{B}}+\mathrm{S}_{\mathrm{B}^{\prime}}\right)^{2} \\
& \mathrm{~S}_{2}=\left(\mathrm{S}_{\mathrm{C}}+\mathrm{S}_{\mathrm{B}^{\prime}}\right)^{2}
\end{aligned}
$$

Sustituimos la ecuación (10), (11) y (12) en las ecuaciones (13) y (14) respectivamente. Se aplica un filtro paso bajo que elimine todos los términos con frecuencias superiores a $f_{f_{1}}\left(f_{r}-f_{f} \approx 10 M H z\right)$; ademas, se elimina la componente continua y se obtiene la expresión simplificada (15) y (16).

$$
\begin{gathered}
\mathrm{S}_{1}=\mathrm{k}_{\mathrm{r}} \mathrm{k}_{\mathrm{L}} \cos \left(2 \pi \mathrm{f}_{\mathrm{I}} \mathrm{t}\right) \\
\mathrm{S}_{2}=\mathrm{k}_{\mathrm{r}} \mathrm{k}_{\mathrm{L}} \cos \left(2 \pi \mathrm{f}_{\mathrm{I}} \mathrm{t}+\phi\right)
\end{gathered}
$$

\section{Balance de Potencias}

En el cuadro 4 se muestran los resultados del balance de potencia, en este tabla se indica el valor de entrada $\left(\mathrm{P}_{\text {in }}\right)$, salida $\left(\mathrm{P}_{\text {out }}\right)$ y de consumo $\left(\mathrm{P}_{\mathrm{cs}}\right)$ de potencia de cada componente, excluyendo a los mezcladores y las antenas.

Basado en la ecuación de transmisión de Friis (17), se calcula las perdidas de potencia entre antenas.

$$
\mathrm{P}_{\mathrm{cs}}=\mathrm{P}_{\mathrm{t}}+\mathrm{D}_{\mathrm{t}}+\mathrm{D}_{\mathrm{r}}+20 \log (4 \pi \mathrm{d} \lambda)
$$

Simulando la propagación por el plasma como si esta fuera una propagación por el vacío y al establecer que ambas antenas son iguales y que poseen una ganancia de $10 \mathrm{~dB}$, se estima que la perdida por propagación es aproximadamente $32.28 \mathrm{~dB}$

Cuadro 4. Balance de Potencias.

\begin{tabular}{|l|c|c|c|}
\hline \multicolumn{1}{|c|}{ Componente } & $\mathrm{P}_{\text {in }}(\mathrm{dBm})$ & $\mathrm{P}_{\text {out }}(\mathrm{dBm})$ & $\mathrm{P}_{\mathrm{cs}}(\mathrm{dBm})$ \\
\hline Osciladores PLL & & 25 & \\
\hline Aisladores & 25 & 23,7 & 1,3 \\
\hline Acoples direccionales & 23,7 & $22,7 / 12,7$ & 1 \\
\hline Atenuador fijo RF & 12,7 & $-7,3$ & 20 \\
\hline Atenuador fijo LO & 22,7 & 12,7 & 10 \\
\hline
\end{tabular}

La potencia de entrada de las señales RF y LO de los mezcladores se presentan en el cuadro 5:

Cuadro 5. Potencia de entrada en los mezcladores.

\begin{tabular}{|l|c|c|}
\hline \multicolumn{1}{|c|}{ Componente } & $\mathrm{RF}(\mathrm{dBm})$ & $\mathrm{LO}(\mathrm{dBm})$ \\
\hline Mezclador S1 & $-7,3$ & 12,7 \\
\hline Mezclador S2 & -9.5 & 12,7 \\
\hline
\end{tabular}




\section{Conclusiones}

Se concluye que el diseño cumple con los requerimientos necesarios para su implementación, permitiendo hacer una comparación simple entre ambas señales $\left(S_{1} y S_{2}\right)$ para determinar el desfase producto de la propagación de la onda en el plasma, y de esta forma mediante la ecuación (8) se calcularía la densidad electrónica experimental. Se recomienda implementar un sistema que digitalice y analice las señales de salida, con el objetivo de obtener un resultado mas preciso. La frecuencia de operación del interferómetro queda sujeta a su implementación, aunque debe quedar claro que esta frecuencia tiene un impacto directo en la sensibilidad y exactitud del sistema.

\section{Referencias}

[1] V.I. Vargas, et al. .Engineering overview of the Fusion Research in Costa Rica: SCR-1 Stellarator and Spherical Tokamak MEDUSA-CR."27th IEEE Symposium on Fusion Engineering (SOFE), Shanghai, China (2017).

[2] T. G. Marques et al, Real-time digital heterodyne interferometer for high resolution plasma density measurements at ISTTOK,ev. Sci. Instrum., vol. 79, (10), pp. 10E711, 2008. Available: https://doi.org/10.1063/1.2956875. DOl: $10.1063 / 1.2956875$.

[3] Solano-Piedra, R., Vargas, V. I., Köhn, A., Coto-Vílchez, F., SánchezCastro, J., López-Rodríguez, D., ...\& Asenjo, J. (2017). Overview of the SCR-1 Stellarator. In 23rd IAEA Technical Meeting on the Research Using Small Fusion Devices.

[4] T. Guerra, Real-Time measurement of the plasma electron density at ISTTOK and study of the ISTTOK hydrogen injection system"Instituto Superior Técnico, Universidad Tecnica de Lisboa , 2008.

[5] J. Iraburu, "Comentarios sobre el interferómetro propuesto para el Stellarator SCR-1." [Inédito]. 2014.

[6] Krämer-Flecken, A.(2006). Microwave and far infrared diagnostics. Transactions of fusion science and technology, 49:387-394. Alemania.

[7] H. J., G. T. and H. M., "Heterodyne methods in millimetre wave plasma diagnostics with applications to ECE, interferometry and reflectometry,ÏOPscience., vol. I, no. PII: S0741-3335(97)61467-0, pp. 4-20, 1997. 